\title{
High level of CFTR expression is associated with tumor aggression and knockdown of CFTR suppresses proliferation of ovarian cancer in vitro and in vivo
}

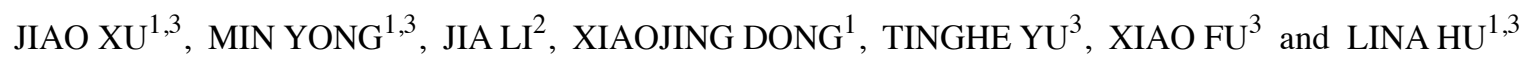 \\ Departments of ${ }^{1}$ Obsterics and Gynecology, ${ }^{2}$ Pathology and ${ }^{3}$ Laboratory of Obsterics and Gynecology, \\ The Second Affiliated Hospital of Chongqing Medical University, Chongqing 400010, P.R. China
}

Received November 17, 2014; Accepted January 5, 2015

DOI: $10.3892 /$ or.2015.3829

\begin{abstract}
The cystic fibrosis transmembrane conductance regulator (CFTR) belongs to the ATP-binding cassette (ABC) transporter family, members of which are involved in various types of cancer. The relationship between CFTR and ovarian cancer remains to be elucidated. The aim of the present study was to investigate the expression of CFTR in human ovarian cancer tissues and its clinical significance in the progression of ovarian cancer. The role of CFTR in the malignant invasion, migration and proliferation of ovarian cancer in vitro and in vivo was also investigated. Immunohistochemical staining analysis was performed to detect the expression of CFTR in 83 cases of human epithelial ovarian cancer specimens. Moreover, SKOV3 and A2780 stable cell lines containing shRNA gene specific for CFTR were established. Cell proliferation and motility were observed and compared with CFTR-RNAi cells. Tumorigenicity of CFTR-RNAi cells was investigated by tumor xenograft experiments conducted subcutaneously in nude mice. The expresssion of CFTR in ovarian cancer was significantly higher than that in benign ovarian tumor and normal ovaries $(\mathrm{P}<0.05)$. In ovarian cancer, CFTR expression was significantly associated with advanced FIGO stage, poor histopathological grade and serum Ca-125 $(\mathrm{P}<0.05)$. Furthermore, we observed that CFTR staining was stronger in the serous type as compared to the other types $(\mathrm{P}<0.05)$. Compared with the negative control, decreased cell invasion, migration, proliferation, adhesion and colony formation were observed in CFTR-RNAi cells in vitro. In vivo, tumorigenic abilities of CFTR-RNAi cells were significantly repressed compared with that of the control groups. CFTR overexpression may play an important role in the development and progression of ovarian cancer. Additionally, the
\end{abstract}

Correspondence to: Dr Lina Hu, Department of Obsterics and Gynecology, The Second Affiliated Hospital of Chongqing Medical University, 76 Linjiang Road, Chongqing 400010, P.R. China

E-mail: cqhulina@hotmail.com

Key words: cystic fibrosis transmembrane conductance regulator, ovarian cancer, RNA interference, tumor progression downregulation of CFTR suppresses aggressive malignant biological behaviors of ovarian cancer cells in vitro and in vivo.

\section{Introduction}

Ovarian cancer is the fifth leading cause of mortality in females, with epithelial ovarian cancer being the most common pathological type (1). In developed countries, it is the most lethal gynecologic cancer since $>70 \%$ of women were diagnosed with advanced stage and cure rates at this stage are $<30 \%$ (2). Identification of molecular markers or pathways may be useful in determining the potential therapeutic targets or novel therapeutic methods.

The cystic fibrosis transmembrane conductance regulator (CFTR), an $170 \mathrm{kDa}$ glycosylated protein, is known as a cAMP-dependent chloride (Cl-) anion conducting channel (3). CFTR is found in epithelial cells of human tissues, including the female reproductive tract (4). By controlling ion and protein transport, CFTR is thought to function in most human cells to assist in the maintenance of cell homeostasis (5). CFTR belongs to the ATP-binding cassette (ABC) transporter family, members of which utilize ATPase activity to transport substrates across cell membranes and which are involved in various types of cancer (6). The CFTR gene mutation, the major of which is the deletion of phenylalanine at position 508, may lead to dysfunction at the plasma membrane (7). Such mutations were associated with an increased risk of digestive tract cancers (8), for example, pancreatic cancer (9). However, it has been demonstrated that, CFTR mutation plays a protective role for the risk of prostate cancer (10), malignant melanoma (7) and lung cancer (11).

In the absence of the CFTR gene mutation, CFTR plays a tumor-suppressing role in regulation of prostate cancer development through miR-193b targeting urokinase plasminogen activator (uPA) (12). Another study proposed that CFTR expression was significantly downregulated in breast cancer, which promotes epithelial-to-mesenchymal transition and is associated with poor prognosis (13). CFTR is also known to be involved in modulating signaling pathways in cell inflammation and apoptosis $(14,15)$. Although previous studies suggested a role for CFTR in various types of cancer, the relationship between CFTR and ovarian cancer remains to be determined. 
Therefore, in the present study, to identify the potential role of CFTR in the pathogenesis of ovarian cancer, CFTR expression was evaluated in human epithelial ovarian cancer, benign epithelial ovarian tumor and normal ovarian tissues using immunohistochemical staining. The role of CFTR in the malignancy of ovarian cancer was observed in CFTR-RNAi SKOV3 and A2780 cells in vitro and in vivo. The present study demonstrated that CFTR expression of ovarian cancer, which was associated with clinical features, was significantly higher than that in benign ovarian tumor and normal ovaries. CFTR knockdown suppressed the malignant behavior of ovarian cancer cells, including cell invasion, motility and proliferation.

\section{Materials and methods}

Patients and specimens. There were 112 paraffin-embedded tissue samples, including 83 epithelial ovarian cancer, 18 benign epithelial tumor and 11 normal ovarian tissues (resected for non-ovarian diseases) that were collected from the Department of Pathology, the Second Affiliated Hospital of Chongqing Medical University between 2010 and 2013. All the ovarian cancer patients underwent surgical exploration and cytoreduction as the initial treatment. The clinicopathological characteristics are presented in Table I. The patients were staged according to the International Federation of Gynecology and Obstetrics (FIGO). The tissue blocks were re-evaluated by two senior pathologists for histological type and histopathologic grading. Tissue samples were obtained following informed consent by the patients. The study protocol was approved by the Ethics Committee of the Second Affiliated Hospital of Chongqing Medical University.

Immunohistochemistry. The tissue slides were deparaffinized in xylene and rehydrated through graded ethanol. Antigen retrieval was performed in $10 \mathrm{mmol} / \mathrm{l}$ boiling sodium citrate buffer at $\mathrm{pH} 6.0$ for $15 \mathrm{~min}$ by microwave irradiation. The slides were then incubated with $3 \%$ hydrogen peroxide $\left(\mathrm{H}_{2} \mathrm{O}_{2}\right)$ for $10 \mathrm{~min}$ at room temperature. After rinsing, the non-specific binding site was blocked with $10 \%$ normal goat serum for $15 \mathrm{~min}$ at room temperature. A mouse monoclonal anti-human CFTR antibody (diluted 1:200, ab2784; Abcam, Cambridge, UK) was applied to slides in a moist chamber at $4^{\circ} \mathrm{C}$ overnight. After washing with phosphate-buffered saline (PBS), the slides were incubated with biotinylated secondary antibody for $15 \mathrm{~min}$ at room temperature (diluted 1:1,000, cat. no. SP-9002; Zhongshan Golden Bridge Inc., China). The slides were treated with $\mathrm{ABC}$ reagent for $15 \mathrm{~min}$ at room temperature, and stained with 3,3'-diaminobenzidine (DAB), followed by counterstaining with hematoxylin.

Cells with brown staining for the membrane and/or cytoplasm were considered positive. A semi-quantitative scoring system was applied to assess protein level based on intensity (0-3: 0 , absence of staining; 1 , weakly stained; 2 , moderately stained; and 3, strongly stained) and percentage of positive tumor cells (0-3: absence of positive cells; $1,0.1-33 \%$ of cells positive; $2,33.1-66 \%$ of cells positive; $3,>66.1 \%$ of cells positive) and generated a score ranging from 0 to 9 when multiplied (16). Low expression was defined as a staining score of $0-4$, and high expression was defined as a staining score of 5-9.
Table I. Association of CFTR expression with clinicopathological characteristics in 83 cases of human epithelial ovarian cancer.

\begin{tabular}{lcccc}
\hline & \multicolumn{4}{c}{ CFTR expression } \\
\cline { 3 - 4 } & $\begin{array}{c}\text { No. of pts } \\
(\mathrm{n}=83)\end{array}$ & $\begin{array}{c}\text { Low no. } \\
(\%)\end{array}$ & $\begin{array}{c}\text { High no. } \\
(\%)\end{array}$ & P-value \\
Characteristics & 47 & $16(34)$ & $31(66)$ & 0.654 \\
Age (years) & 36 & $14(39)$ & $22(61)$ & \\
$<51.0$ & & & & \\
$>51.0$ & & & & \\
Serum Ca-125 & 17 & $11(65)$ & $6(35)$ & $0.010^{\mathrm{a}}$ \\
level (U/ml) & 66 & $19(29)$ & $47(71)$ & \\
$<35$ & & & & \\
$>35$ & 31 & $18(58)$ & $13(42)$ & $0.002^{\mathrm{a}}$ \\
FIGO stage & 52 & $12(23)$ & $40(77)$ & \\
I/II & & & & \\
III/IV & & & & \\
Histological grade & & & & \\
1 & 18 & $14(78)$ & $4(22)$ & $0.000^{\mathrm{a}}$ \\
2 & 29 & $9(31)$ & $20(69)$ & \\
3 & 36 & $7(19)$ & $29(81)$ &
\end{tabular}

Grades $2-3$ vs. 1

Ascites (ml)

$<100$
$>100$

0.384

Tumor type

Serous

Mucinous

Clear cell

Endometrioid

compared with

serous type

23 (34) 45 (66)

Tumor size $(\mathrm{cm})$

$\begin{array}{rrrrr}<5 & 13 & 2(15) & 11(85) & 0.121 \\ >5 & 70 & 28(40) & 42(60) & \end{array}$

Serum HE4

level (pm)

$\begin{array}{lllll}<70 & 22 & 11(50) & 11(50) & \\ >70 & 61 & 19(31) & 42(69) & 0.129\end{array}$

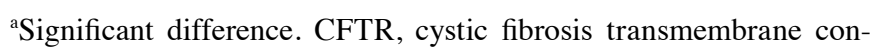
ductance regulator; pts, patients.

Cell lines and culture conditions. The human A2780 and SKOV3 epithelial ovarian cancer cell lines were obtained from the Laboratory of Obsterics and Gynecology, the Second Affiliated Hospital of Chongqing Medical University. The cells were cultured in Dulbecco's modified Eagle's medium supplemented with $10 \%$ fetal bovine serum and antibiotics (100 U/ml penicillin and $100 \mu \mathrm{g} / \mathrm{ml}$ streptomycin) (all from Invitrogen, Carlsbad, CA, USA). The cells were cultured in an incubator with $5 \% \mathrm{CO}_{2}$ at $37^{\circ} \mathrm{C}$. 
Western blotting. Total proteins were extracted using lysis buffer (Beyotime, Jiangsu, China) consisting of $50 \mathrm{mM}$ Tris (pH 7.4), 1\% Triton X-100, $1 \%$ sodium deoxycholate, $0.1 \%$ SDS and a protease inhibitor mixture supplemented with $1 \mathrm{mM}$ phenylmethanesulfonyl fluoride (PMSF) according to the manufacturer's instructions. The protein concentration was measured using a BCA protein assay kit (Beyotime). Total proteins $(100 \mu \mathrm{g})$ of each sample were separated on $8 \%$ SDS-PAGE gel by electrophoresis and transferred to polyvinylidene fluoride (PVDF) membranes (Millipore Corporation, Billerica, MA, USA). The membranes were then blocked in $5 \%$ skim milk at $37^{\circ} \mathrm{C}$ for $1 \mathrm{~h}$. Subsequently, the membranes were incubated, respectively, in mouse monoclonal anti-human CFTR antibody (diluted 1:1,000, ab2784) and rabbit monoclonal anti- $\beta$-actin antibody (diluted 1:1,000, ab133626) (both from Abcam) at $4^{\circ} \mathrm{C}$ overnight. After washing with TBST, the membranes were incubated with HRP-conjugated secondary antibodies for $1 \mathrm{~h}$ at room temperature. Proteins were visualized with the ECL system (GE Healthcare, Pittsburgh, PA, USA) using the ChemiDoc XRS system (Bio-Rad, Philadelphia, PA, USA).

Gene knockdown. To knockdown CFTR in the ovarian cells, four human CFTR-specific small hairpin RNA (shRNA) expression vectors in pGFP-V-RS plasmid were purchased from OriGene (TG313958; OriGene Technologies, Inc., Rockville, MD, USA). Cells transfected with non-effective shRNA cassette in pGFP-V-RS plasmid were used as a negative control. Vector DNA $(1 \mu \mathrm{g})$ was transfected into A2780 and SKOV3 cells by PolyJet ${ }^{\mathrm{TM}}$ transfection reagent (SignaGen Laboratories, Ijamsville, MD, USA). At two days after transfection, $2.5 \times 10^{6}$ cells were digested and replanted into five $10-\mathrm{cm}$ dishes in full medium containing puromycin at concentration of $0.9 \mu \mathrm{g} / \mathrm{ml}$ for A2780 and $0.5 \mu \mathrm{g} / \mathrm{ml}$ for SKOV3 cells, and selected for 2-3 weeks. Puromycin-resistant and GFP-positive cell colonies were separated and verified by western blotting. The stably transfected cell lines were then cultured in medium containing $0.5 \mu \mathrm{g} / \mathrm{ml}$ of puromycin for A2780 and $0.25 \mu \mathrm{g} / \mathrm{ml}$ for SKOV3 cells for subsequent studies.

Cell invasion and migration assays. For cell invasion and migration assays, a cell invasion assay kit $(8-\mu \mathrm{m}$ pore size; Cell Biolabs Inc., San Diego, CA, USA) was used according to the manufacturer's instructions. Approximately $1 \times 10^{5}$ cells were placed into the Transwell insert with serum-free media. After 24-48 h, the cells on the inner layer of the Transwell insert were wiped away, then inserts were fixed and stained, and photographed with a light microscope under high magnification objective. Each insert was then transferred to an empty well, and extracted with extraction solution for $10 \mathrm{~min}$ on an orbital shaker. Extraction solution $(100 \mu \mathrm{l})$ from each sample was transferred to a 96-well microtiter plate and OD $560 \mathrm{~nm}$ was measured using a microplate reader (Thermo Scientific, Waltham, MA, USA). The procedure was repeated independently three times with triplicate inserts for each group.

Plate colony formation assay. We seeded 200 cells into 6-well culture plates in triplicate. The cells were collected after 14 days and then stained with Giemsa stain. The colony containing $>50$ cells was counted under the microscope.
Cell adhesion assay. We pre-coated 96-well plates with $100 \mu \mathrm{l}$ Matrigel at concentration of $0.04 \mu \mathrm{g} / \mathrm{ml}$ (BD Matrigel, Franklin Lakes, NJ, USA). Prior to cell seeding, the plates were incubated in serum-free medium at $37^{\circ} \mathrm{C}$ for $30 \mathrm{~min}$ for rehydration. Cells $\left(3 \times 10^{4}\right)$ were seeded in $100 \mu 1$ growth medium in triplicate and incubated for $1 \mathrm{~h}$. After rinsing, the adherent cells were fixed using $4 \%$ formaldehyde for $10 \mathrm{~min}$, and stained by crystal violet. Adherent cell staining was extracted by acetic acid and quantified using a microplate reader (Thermo Scientific) at OD $490 \mathrm{~nm}$.

Cell proliferation assay. For the proliferation assay, 500 cells were seeded in 96-well plates in triplicate and cultured for days 1, 2, 3, 4 and 5. The ST-8 Cell Counting Kit (KeyGen Biotech, Nanjing, China) and a modified 3-(4,5-dimethylthiazol-2-yl)2,5-diphenyltetrazolium bromide (MTT) assay method, were applied to estimate the proliferation of viable cells. The cells were collected at specific time-points $(6,24,48,72,96$ and $120 \mathrm{~h}$ ) and incubated for an additional $2 \mathrm{~h}$ in $10 \%$ WST- 8 solution at $37^{\circ} \mathrm{C}$. Absorbance was measured at $450 \mathrm{~nm}$ using a microplate reader (Thermo Scientific). The procedure was repeated independently three times.

Tumor formation in nude mice. To investigate tumorigenicity, 5-week-old athymic female nude mice were provided by the Institution Animal Care Committee at Chongqing Medical University. Approximately $4 \times 10^{6}$ cells in $100 \mu 1$ suspension of SKOV3-CFTRi cells (7 implants) with PBS and SKOV3-NC cells (cells transfected with non-effective shRNA cassette) as a control (7 implants) were injected subcutaneously. The tumor size was measured on a weekly basis and monitored over 5 weeks. At the end of the experiment, the mice were euthanized and the xenografts collected for subsequent analysis. The tumor size was calculated as: $0.5234 \mathrm{x}$ [long diameter (short diameter) $\left.{ }^{2}\right]$. The experimental procedure was approved by the Institution Animal Care Committee at Chongqing Medical University.

Statistics analysis. Independent Student's t-test was used to make a comparison of the data between two groups. Relevance analysis between CFTR immunostaining and clinicopathological characteristics was performed by Pearson's $\chi^{2}$ or Fisher's exact tests. Statistical analysis was carried out by SPSS 17.0 software (SPSS, Inc., Chicago, IL, USA). P<0.05 (two-sided) was considered to indicate a statistically significant result.

\section{Results}

CFTR exhibits a high expression in ovarian cancer and is correlated with clinicopathological characteristics. CFTR staining was predominantly detected in the cell membrane and cytoplasm (Fig. 1A). The expression of CFTR in ovarian cancer specimens was significantly higher than that in benign $(\mathrm{P}<0.05)$ and normal ovaries $(\mathrm{P}<0.05$, Fig. 1B). Moreover, in a majority of benign tumors (77.8\%) and normal ovaries (90.1\%), it was difficult to detect CFTR, which was in contrast to the cancer group (Fig. 1B). Regarding the clinicopathological characteristics, the statistical analysis revealed that the CFTR protein level was well-related to advanced clinical 


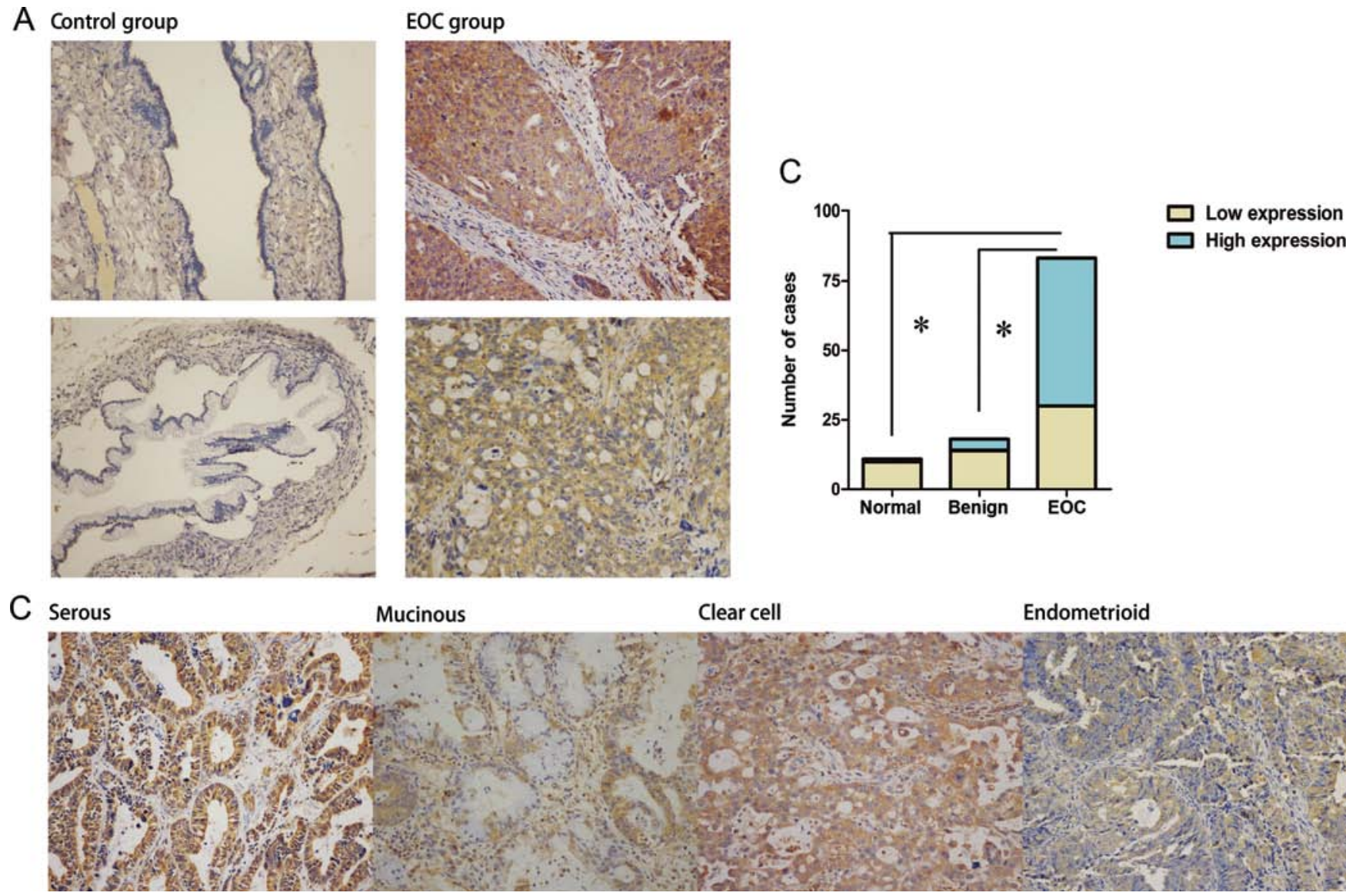

Figure 1. Expression of CFTR in epithelial ovarian cancer, benign ovarian tumor and normal ovary tissue samples. (A and B) By immunohistochemical staining, CFTR staining was predominantly localized in the cytoplasm and cytomembrane of epithelial ovarian cancer tissues ( $\mathrm{n}=83$ ). By contrast, CFTR expression was undetectable in a majority of benign $(n=18)$ samples and normal ovaries $(n=11)$ (original magnification, $x 200)$. $P<0.05$. (C) CFTR was expressed in four different histological types of epithelial ovarian cancer samples (original magnification, x200). CFTR, cystic fibrosis transmembrane conductance regulator.

stages (stage III/IV vs. I/II, P<0.05), poor histological grade (grade $2-3$ vs. $1, \mathrm{p}<0.05$ ), and a higher serum Ca-125 level $(\mathrm{P}<0.05$, Table I). Furthermore, we observed that CFTR staining was stronger in the serous type and clear cell type as compared to the remaining types $(\mathrm{P}<0.05$, Table I).

Silencing of CFTR expression by RNA interference. To examine the role of CFTR in serous ovarian cancer cells, we knocked down CFTR in SKOV3 and A2780 cells. The cells were transfected with CFTR-specific shRNA expression pGFP-V-RS vectors and the stably transfected cells were selected (referred to as CFTRi in Fig. 2A and B). Non-effective shRNA-infected cells were designated as a negative control, referred to as SKOV3-NC and A2780-NC. Western blot results revealed that the CFTR protein level of CFTRi cells was significantly reduced compared with that of its corresponding control cells (Fig. 2C). Thus, SKOV3-CFTRi3 and A2780-CFTRi2 were used in the subsequent experiments.

Knockdown of CFTR inhibits cell motility and invasion in vitro. SKOV3-CFTRi3 cells exhibited an impaired migration capacity of $60.89 \%$ compared to SKOV3-NC cells $(\mathrm{P}<0.05)$ (Fig. 3A and B). Similarly, A2780-CFTRi2 cells had a reduced migration capacity of $63.93 \%$ compared to A2780-NC cells $(\mathrm{P}<0.05)$. For SKOV3-CFTRi3 and A2780-CFTRi2, an $\sim 41.72$ and $63.11 \%$ reduction in the number of migratory cells was observed compared with SKOV3-NC and A2780-NC cells ( $\mathrm{P}<0.05$ for both, Fig. 3C and D).

Knockdown of CFTR inhibits cell adhesion in vitro. As shown in Fig. 3E, there was a 42.31 and $38.52 \%$ decreased percentage of optical density (OD) value in SKOV3-CFTRi3 and A2780-CFTRi2 cells compared to the control cells $(\mathrm{P}<0.05$ for both).

Knockdown of CFTR inhibits proliferation and colony formation in vitro. The results of the WST-8 assay (Fig. 4C and D) showed that the growth ability of SKOV3-CFTRi3 and A2780-CFTRi2 cells decreased compared with the corresponding control cells. Moreover, CFTR knockdown cells also had a low colony formation ability as the number of colonies of CFTRi cells were decreased when compared with the corresponding control cells (Fig. 4A and B).

CFTR knockdown inhibits xenograft tumor formation. Cells were subcutaneously injected into the flanks to form xenograft tumors in nude mice. We measured the size of xenograft tumors once a week for a total of five weeks. We observed that the tumor size of the SKOV3-CFTRi3 group was statistically significantly smaller than that of the SKOV3-NC group from 
A $\mathbf{A} 2780$

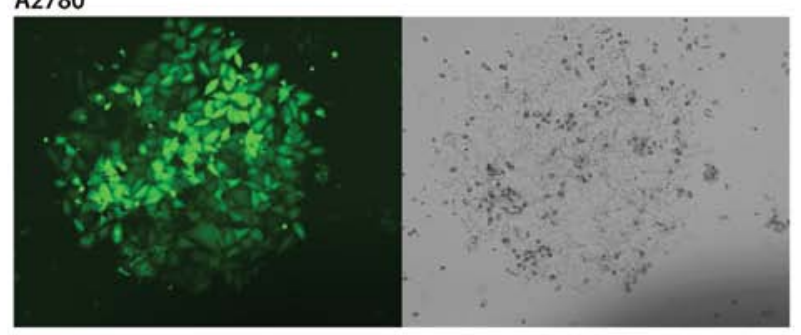

SKOV3

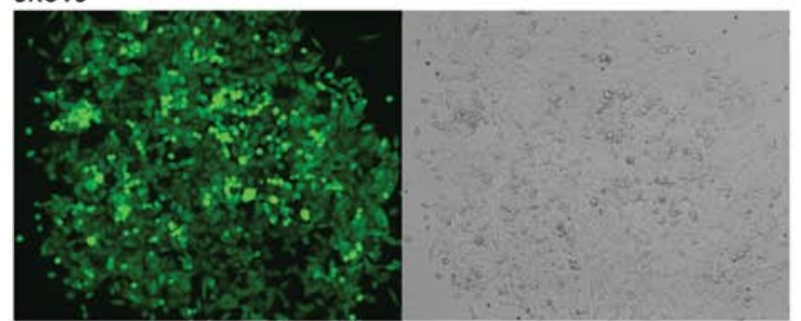

C

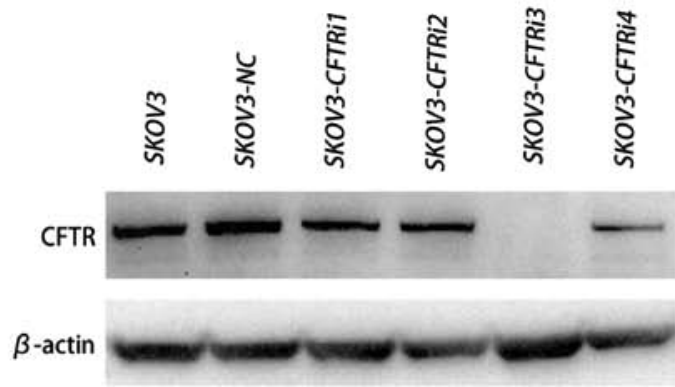

B
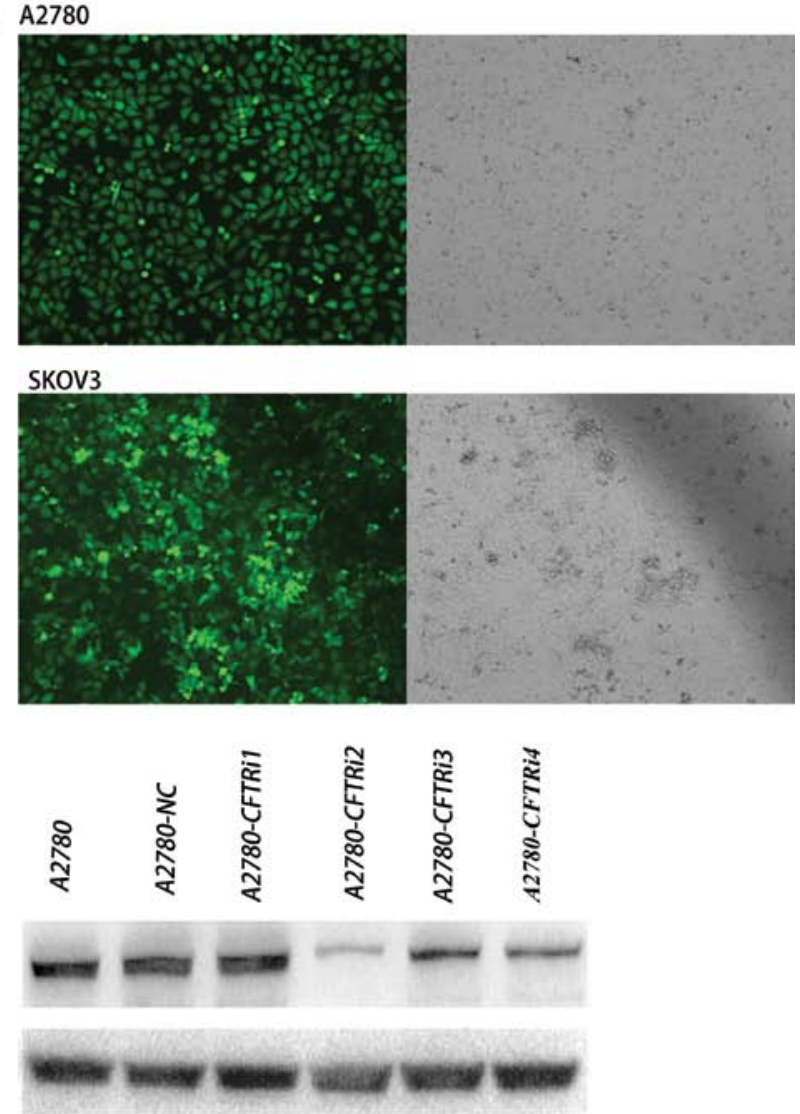

Figure 2. Human A2780 and SKOV3 epithelial ovarian cancer cell lines were transfected with CFTR-specific shRNA expression pGFP-V-RS vectors and the stably transfected cells were selected. (A) Strong green fluorescence was detected under fluorescence microscopy after 2-3 weeks selection with puromycin at a concentration of $0.9 \mu \mathrm{g} / \mathrm{ml}$ for A2780 and $0.5 \mu \mathrm{g} / \mathrm{ml}$ for SKOV3 cells, respectively (original magnification, x100). (B) Strong green fluorescence was also detected under fluorescence microscopy after 2 months selection with puromycin at a concentration of $0.5 \mu \mathrm{g} / \mathrm{ml}$ for A2780 and $0.25 \mu \mathrm{g} / \mathrm{ml}$ for SKOV3 cells (original magnification, $\mathrm{x} 100$ ). (C) The CFTR protein of each puromycin-resistant cell as well as GFP-positive cell colonies and negative control cells was measured by western blot analysis $(\sim 170 \mathrm{kDa})$. $\beta$-actin was used as an internal control $(\sim 43 \mathrm{kDa})$. Representative images are shown. CFTR, cystic fibrosis transmembrane conductance regulator.

the third week until the end of the experiment (Fig. 5A). The size of xenograft tumors of SKOV3-CFTRi3 $\left(183 \pm 61 \mathrm{~cm}^{3}\right)$ was significantly smaller than that of the control $\left(713 \pm 270 \mathrm{~cm}^{3}\right)$ at the end of experiment (Fig. 5B and C). At the same time, the average tumor weight of SKOV3-CFTRi3 group was lower than that of the control group (Fig. 5D). Notably, these results showed that CFTR knockdown affected the tumor size significantly rather than the incidence of tumor formation, suggesting that CFTR knockdown suppresses tumor progression rather than tumor initiation in vivo.

\section{Discussion}

CFTR is a membrane of the ATP-binding cassette (ABC) transporter family, which is an ancient family of transmembrane proteins. The ABCC subfamily of $\mathrm{ABC}$ transporters includes CFTR and the MRPs, which were active drug exporters (6). In this study, we initially investigated the expression and localization of CFTR in ovarian cancer, benign tumors and normal ovaries by immunohistochemical analysis. The results showed that CFTR expression was significantly increased in ovarian cancer compared with the other tissue types. Moreover, we focused on the association between the CFTR and clinicopathological characteristics to determine the potential clinical significance in ovarian cancer. Of those characteristics, the high expression of CFTR was well-associated with poor clinical stage, advanced histological grade and an increased Ca-125 level. These results indicated that the expression of CFTR had a positive correlation with the progression state and malignancy degree of ovarian cancer, suggesting that CFTR is a potential biomarker in progression of ovarian cancer. In subsequent experiments, we investigated the expression of CFTR in metastasis outside the pelvis or retroperitoneal or inguinal node of patients, and measured the conditions of prognosis and survival data of patients to evaluate the prognostic value of CFTR. Notably, we found that CFTR expression was significantly higher in the serous carcinoma and clear cell carcinoma as compared to the remaining carcinomas used in our study. Serous ovarian cancer is the most common histotype and accounts for more than half of epithelial ovarian cancer patients (17). Therefore, we selected the serous ovarian cancer cell lines for further study.

In our experiments, the expression of CFTR in SKOV3 and A2780 cells was knocked down by shRNA-mediated gene silencing. To enhance the efficiency of transfection, we generated and isolated stable transfectants proved important in the investigation of the function of CFTR in malignant behaviors in vivo. It was observed that CFTR-RNAi cells showed 
A SKOV3-NC

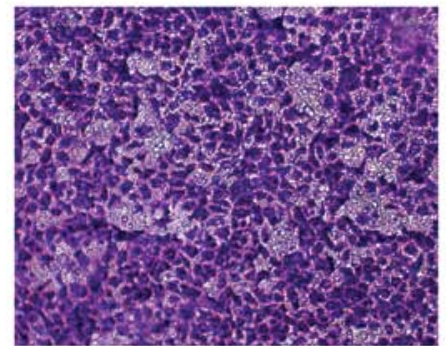

A2780-NC

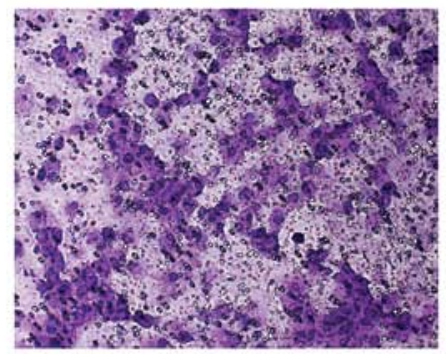

C

SKOV3-NC

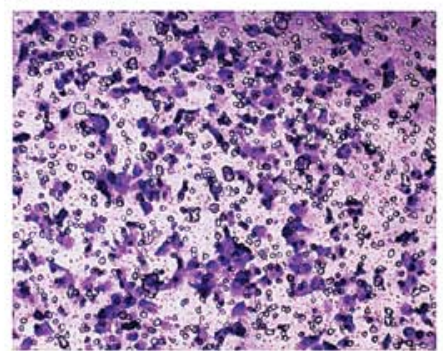

A2780-NC

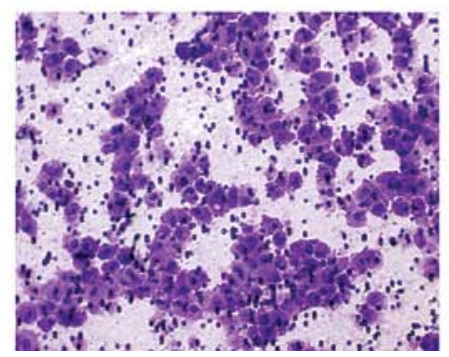

SKOV3-CFTRi

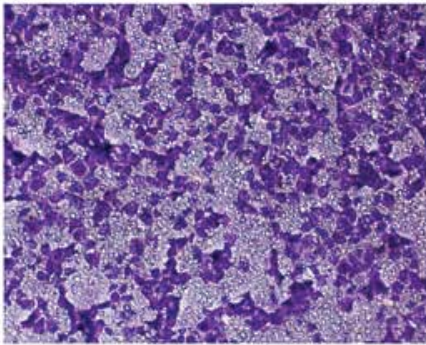

A2780-CFTRi

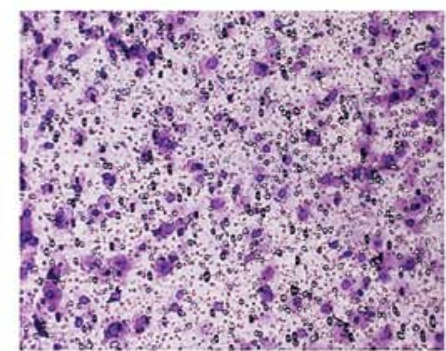

SKOV3-CFTRi

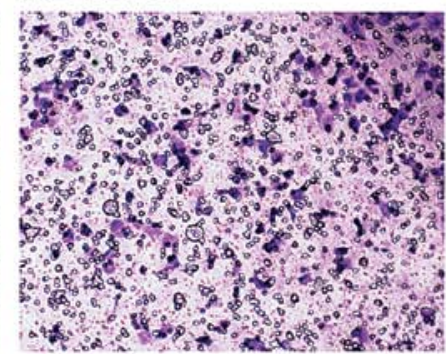

A2780-CFTRi

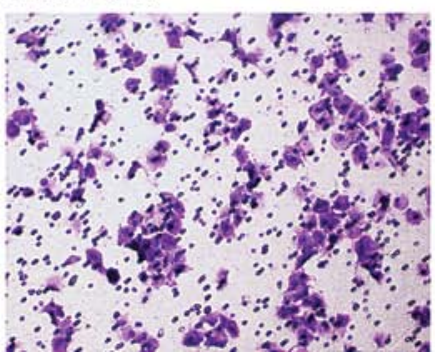

B

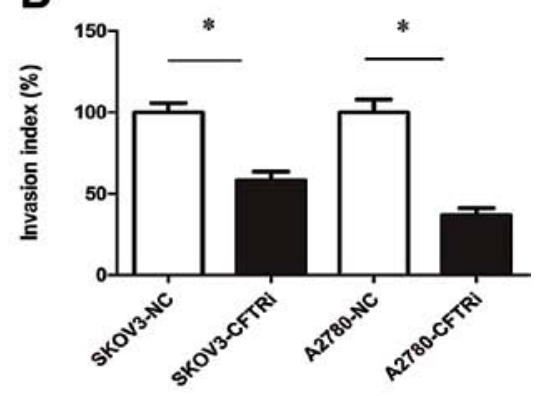

D

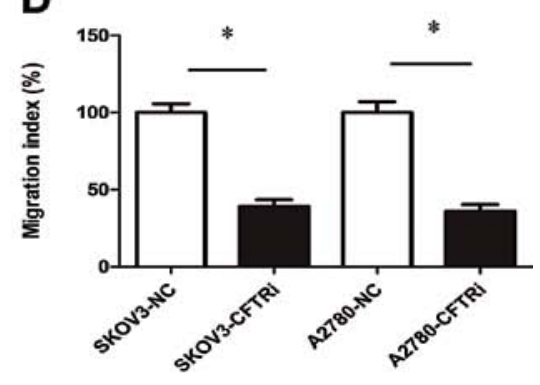

E

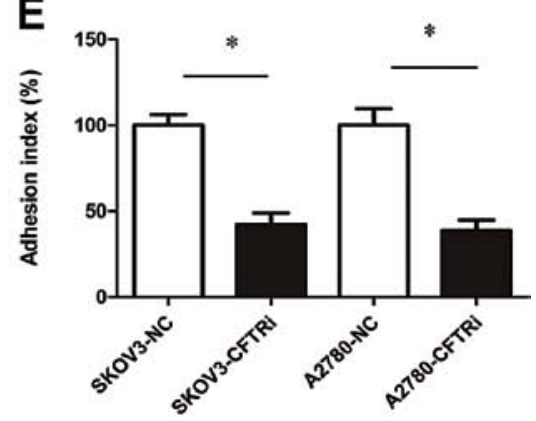

Figure 3. Knockdown of CFTR inhibits cell invasion, migration and cell adhesion in vitro. (A and B) Invasion assays showed an impaired capacity compared with the control cells in the invasion assay, $\mathrm{P}<0.05$. (C and D) SKOV3-CFTRi and A2780-CFTRi cells exhibit a marked impaired capacity compared with the control cells in the migration assays, $\mathrm{P}<0.05$. (E) SKOV3-CFTRi and A2780-CFTRi cells exhibit a marked impaired capacity compared with the control cells in the adhesion assays, $\mathrm{P}<0.05$. CFTR, cystic fibrosis transmembrane conductance regulator.

decreased cell motility, invasion, adhersion, colony formation, and proliferation, particularly migration and invasion. We have demonstrated the effect of CFTR knockdown on the inhibition of malignant behaviors, indicating that CFTR is a potential therapeutic target to ovarian cancer treatment.

We also investigated the function of CFTR in malignant behaviors in vivo. The results revealed xenograft tumors existing in CFTR expressed larger tumor size and rapid tumor growth. The present study has therefore demonstrated that inhibition of CFTR suppressed xenograft tumor development in vivo.

Accumulating evidence suggests that CFTR plays a key role in the progression and metastasis of cancer. Genetic variations in CFTR may be associated with increased or decreased risk for developing cancers, suggesting that CFTR is a tumor suppressor or promoter, according to the type of tumor (7,9-11), MUC4, an important protein suppressing the progression and metastasis of pancreatic cancer cells, has proven to be downregulated by CFTR (18). In the female reproductive system, it has been reported that the overexpression of CFTR in cervical cancer is concerned with cancer development, aggressive biological behaviors and poor prognosis of patients (19).

Previous studies have showed that CFTR binds to ezrinradixin-moesin-binding (ERM) phosphoprotein 50 (EBP50), an apical membrane PDZ domain-containing protein. EBP50 is essential in the apical polarization of CFTR in epithelial cells (20) and is known to interact via its C-terminal domain with the ERM-family proteins, which in turn bind to the actin cytoskeleton (21). We hypothesized that the CFTR affects 
A
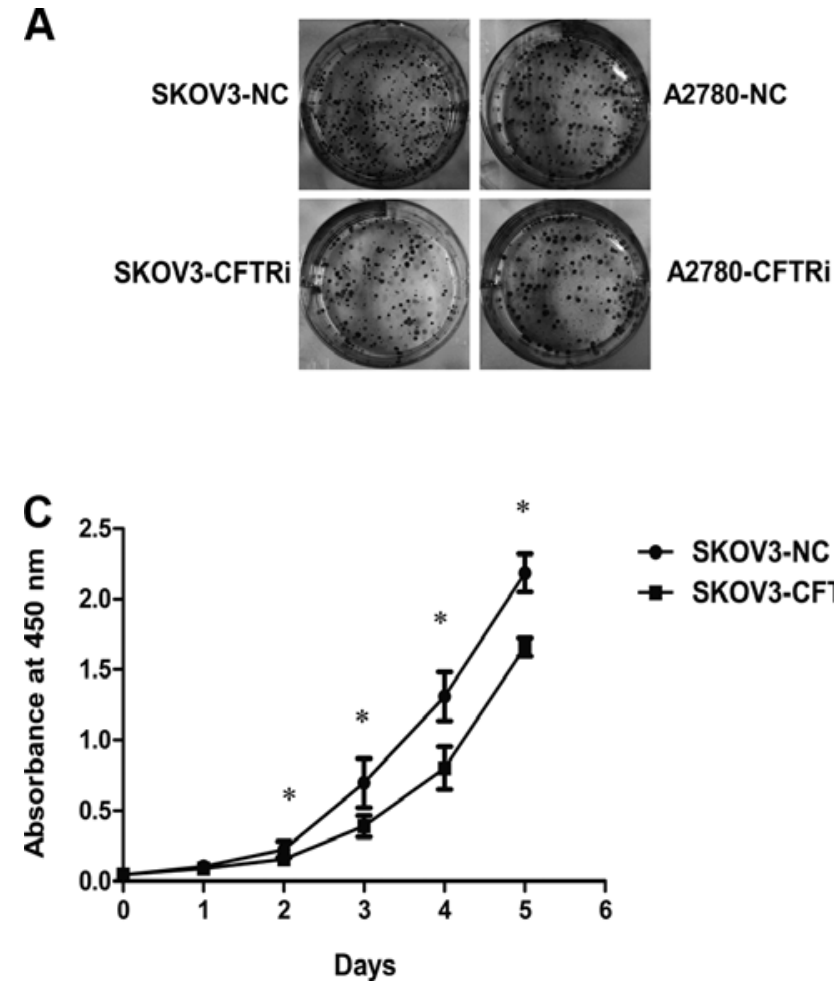

B

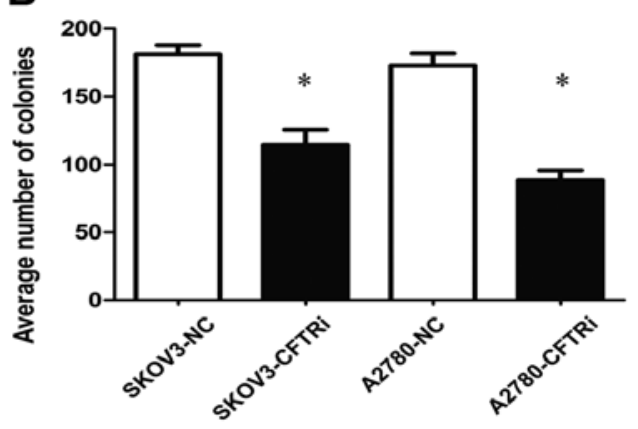

Figure 4. Knockdown of CFTR inhibits colony formation and proliferation in vitro. (A and B) Statistical analysis of the colony-formation assay is shown. The number of CFTRi cell colonies was decreased compared with it corresponding control cells; P<0.05. (C and D) As evaluated using MTT assay, SKOV3-CFTRi and A2780-CFTRi cells demonstrated a significant growth-inhibiting effect from the second day compared with the control cells. CFTR, cystic fibrosis transmembrane conductance regulator.

A

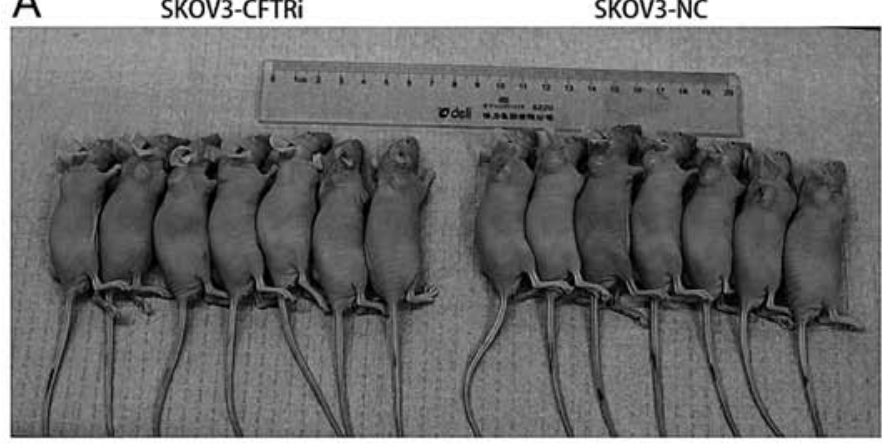

C

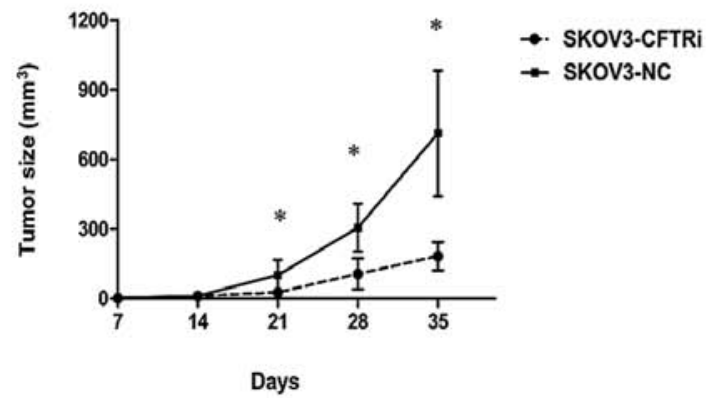

B

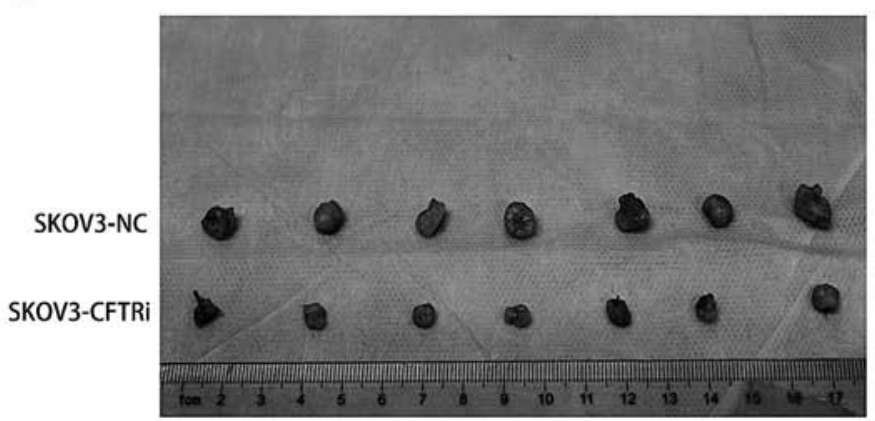

D

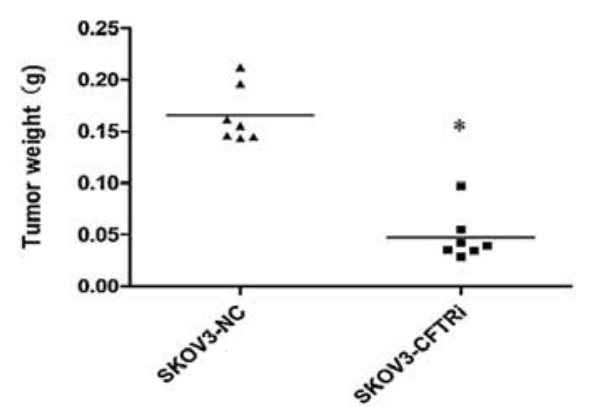

Figure 5. CFTR knockdown inhibits xenograft tumor formation in vivo. (A and B) Images of mice and tumor samples at the fifth week after SKOV3-CFTRi and SKOV3-NC cell injection. (C) Tumor growth curves of xenografts in nude mice. (D) Tumor weight of group SKOV3-CFTRi and SKOV3-NC, P<0.05. CFTR, cystic fibrosis transmembrane conductance regulator.

the cell adhersion, invasion and migration abilities due to connecting membrane rafts to the actin cytoskeleton.
However, EBP50 is identified as a specific Cbp-binding partner, and $\mathrm{Cbp}$ binds the protein tyrosine kinase Csk (21). The 
Cbp-Csk complex suppresses the activity of membrane-associated Src-family kinases which play an important role in the regulation of essential cell functions such as growth and receptor signaling (22-24). Therefore, we suggest that the overexpression of CFTR in ovarian cancer progression may be associated with the activation of c-src signaling pathway. Thus, further investigation is needed to identify the mechanisms of CFTR in ovarian cancer development and progression.

In conclusion, to the best of our knowledge, our data have provided clinical and laboratorial evidence for the first time that CFTR expression is well correlated with ovarian cancer progression and aggressive behaviors, indicating CFTR may be a novel tumor marker for ovarian cancer, particularly for serous carcinoma. However, a greater number of cases should be included to make the prospective studies valid. Nevertheless, our result supports the important role of CFTR in ovarian cancer.

\section{Acknowledgements}

This study was supported by the National Natural Science Foundation of China (grant no. 81172492), the Key Project of Chongqing Science and Technology Commission (grant no. CSTC 2012JJB10030), and the Key Project of Chongqing Municipal Health Bureau (grant no. 2011-1-056).

\section{References}

1. Siegel R, Naishadham D and Jemal A: Cancer statistics, 2013 CA Cancer J Clin 63: 11-30, 2013.

2. Rauh-Hain JA, Krivak TC, Del Carmen MG and Olawaiye AB: Ovarian cancer screening and early detection in the general population. Rev Obstet Gynecol 4: 15-21, 2011.

3. Bear CE, Li CH, Kartner N, et al: Purification and functional reconstitution of the cystic fibrosis transmembrane conductance regulator (CFTR). Cell 68: 809-818, 1992.

4. Tizzano EF, Silver MM, Chitayat D, Benichou JC and Buchwald M: Differential cellular expression of cystic fibrosis transmembrane regulator in human reproductive tissues. Clues for the infertility in patients with cystic fibrosis. Am J Pathol 144: 906-914, 1994

5. Schwiebert EM, Benos DJ,Egan ME, Stutts MJ and Guggino WB CFTR is a conductance regulator as well as a chloride channel. Physiol Rev 79 (Suppl 1): S145-S166, 1999.

6. Higgins $\mathrm{CF}$ : $\mathrm{ABC}$ transporters: from microorganisms to man Annu Rev Cell Biol 8: 67-113, 1992.

7. Padua RA, Warren N, Grimshaw D, et al: The cystic fibrosis $\Delta$ F508 gene mutation and cancer. Hum Mutat 10: 45-48, 1997.

8. Neglia JP, FitzSimmons SC, Maisonneuve P, et al: The risk of cancer among patients with cystic fibrosis. Cystic Fibrosis and Cancer Study Group. N Engl J Med 332: 494-499, 1995.
9. McWilliams RR, Petersen GM, Rabe KG, et al: Cystic fibrosis transmembrane conductance regulator (CFTR) gene mutations and risk for pancreatic adenocarcinoma. Cancer 116: 203-209, 2010.

10. Qiao D, Yi L, Hua L, et al: Cystic fibrosis transmembrane conductance regulator $(C F T R)$ gene $5 \mathrm{~T}$ allele may protect against prostate cancer: a case-control study in Chinese Han population. J Cyst Fibros 7: 210-214, 2008.

11. Li Y, Sun Z, Wu Y, et al: Cystic fibrosis transmembrane conductance regulator gene mutation and lung cancer risk. Lung Cancer 70: 14-21, 2010.

12. Xie C, Jiang XH, Zhang JT, et al: CFTR suppresses tumor progression through miR-193b targeting urokinase plasminogen activator (uPA) in prostate cancer. Oncogene 32: 2282-2291, 2291.e1-2291.e7, 2013.

13. Zhang JT, Jiang XH, Xie C, et al: Downregulation of CFTR promotes epithelial-to-mesenchymal transition and is associated with poor prognosis of breast cancer. Biochim Biophys Acta 1833: 2961-2969, 2013

14. Wu Z, Peng X, Li J, Zhang Y and Hu L: Constitutive activation of nuclear factor $\kappa \mathrm{B}$ contributes to cystic fibrosis transmembrane conductance regulator expression and promotes human cervical cancer progression and poor prognosis. Int J Gynecol Cancer 23: 906-915, 2013.

15. Jacquot J, Tabary O, Le Rouzic P and Clement A: Airway epithelial cell inflammatory signalling in cystic fibrosis. Int $\mathrm{J}$ Biochem Cell Biol 40: 1703-1715, 2008.

16. Au CW, Siu MK, Liao X, et al: Tyrosine kinase B receptor and BDNF expression in ovarian cancers - effect on cell migration, angiogenesis and clinical outcome. Cancer Lett 281: 151-161, 2009.

17. Wang J, Dai JM, Che YL, et al: Elmo1 helps dock180 to regulate Racl activity and cell migration of ovarian cancer. Int J Gynecol Cancer 24: 844-850, 2014.

18. Singh AP, Chauhan SC, Andrianifahanana M, et al: $M U C 4$ expression is regulated by cystic fibrosis transmembrane conductance regulator in pancreatic adenocarcinoma cells via transcriptional and post-translational mechanisms. Oncogene 26: 30-41, 2007.

19. Peng X, Wu Z, Yu L, et al: Overexpression of cystic fibrosis transmembrane conductance regulator (CFTR) is associated with human cervical cancer malignancy, progression and prognosis. Gynecol Oncol 125: 470-476, 2012.

20. Moyer BD, Denton J, Karlson KH, et al: A PDZ-interacting domain in CFTR is an apical membrane polarization signal. $\mathbf{J}$ Clin Invest 104: 1353-1361, 1999.

21. Brdicková N, Brdicka T, Andera L, et al: Interaction between two adapter proteins, PAG and EBP50: a possible link between membrane rafts and actin cytoskeleton. FEBS Lett 507: 133-136, 2001.

22. Wiener JR, Windham TC, Estrella VC, et al: Activated Src protein tyrosine kinase is overexpressed in late-stage human ovarian cancers. Gynecol Oncol 88: 73-79, 2003.

23. Wiener JR, Nakano K, Kruzelock RP, Bucana CD, Bast RC Jr and Gallick GE: Decreased Src tyrosine kinase activity inhibits malignant human ovarian cancer tumor growth in a nude mouse model. Clin Cancer Res 5: 2164-2170, 1999.

24. Kim HS, Han HD, Armaiz-Pena GN, et al: Functional roles of Src and Fgr in ovarian carcinoma. Clin Cancer Res 17: 1713-1721, 2011. 